\title{
Influence of nanoencapsulated lutein on acetylcholinesterase activity: In vitro determination, kinetic parameters, and in silico docking simulations
}

\author{
Cristiane Grella Miranda ${ }^{b}$, Priscila Dayane Freitas dos Santos ${ }^{\mathrm{a}}$, Jéssica Thaís do Prado Silva ${ }^{\mathrm{a}}$, \\ Fernanda Vitória Leimann ${ }^{\mathrm{b}, \mathrm{c}, \mathrm{d}}$, Bianca Ferreira Borges ${ }^{\mathrm{b}, \mathrm{d}}$, Rui Miguel Abreu ${ }^{\mathrm{d}}$, Rafael Porto Ineu ${ }^{\mathrm{b}}$, \\ Odinei Hess Gonçalves ${ }^{\mathrm{b}, \mathrm{c}, \mathrm{d}}$
}

\author{
a Post-Graduation Program of Food Technology (PPGTA), Federal University of Technology - Paraná - UTFPR, Campus Campo Mourão, via Rosalina Maria dos Santos, \\ 1233, CEP 87301-899, Caixa Postal: 271, Campo Mourão, PR, Brazil \\ ${ }^{\mathrm{b}}$ Food Department (DALIM), Federal University of Technology - Paraná - UTFPR, Campus Campo Mourão, via Rosalina Maria dos Santos, 1233, CEP 87301-899, Caixa \\ Postal: 271, Campo Mourão, PR, Brazil \\ ${ }^{\mathrm{c}}$ Laboratory of Separation and Reaction Engineering - Laboratory of Catalysis and Materials (LSRE-LCM), Polytechnic Institute of Bragança, Campus Santa Apolónia, \\ 1134, 5301-857 Bragança, Portugal \\ ${ }^{\mathrm{d}}$ Centro de Investigação de Montanha (CIMO), Instituto Politécnico de Bragança, Campus Santa Apolónia, 5300-253 Bragança, Portugal
}

\section{A R T I C L E I N F O}

\section{Keywords:}

Nutraceutical

Cholinergic hypothesis

Encapsulation

Nanotechnology

Bioactive

\begin{abstract}
A B S T R A C T
Lutein is a bioactive found in dark leafy vegetables that may be used as a nutraceutical agent in foodstuff and an inhibitor of key enzymes of the human body such as those involved in the cholinergic system. However, its high hydrophobicity leads to low bioavailability and must be overcome if lutein is to be added in foods. The objective of this study was to evaluate the influence of nanoencapsulated lutein in the activity of the acetylcholinesterase enzyme. The in vitro study was carried out using water in order to evaluate the impact of encapsulation on the hydrophilicity of lutein. In vitro assays showed that lutein, both free and nanoencapsulated, presented a mixedtype inhibition behavior, and encapsulated lutein was able to inhibit acetylcholinesterase activity even in an aqueous medium. Inhibition was also showed by the in silico docking results which show that lutein interacted with the pocket region of the enzyme.
\end{abstract}

\section{Introduction}

Lutein is a xanthophyll carotenoid that has attracted the attention of the food industry due to its various biological properties such as antioxidant, anti-inflammatory, and neuroprotective effects. It is not synthesized by the organism and all lutein present in the body must come from dietary sources. The main natural sources of lutein are dark leafy vegetables, most notably spinach and kale (Alves-Rodrigues \& Shao, 2004; Kim, Clark, Park, Lee, \& Fernandez, 2012; Li, Turner, \& Brautigan, 2015). It is found accumulated in the macular region of the retina and is involved in the protection of the photoreceptors against oxidative damage (Kim et al., 2012; Lakshminarayana et al., 2008). Lutein deficiency is associated with cases of degenerative macular, retinal inflammatory reaction, and oxidative stress (Kim et al., 2012).

Although the use of lutein as a supplement or an ingredient in nutraceutical foods is increasing, lutein presents some drawbacks such as its low stability and especially its hydrophobicity. The latter is a major problem for the industrial use of lutein since most foods are waterbased and also because bioavailability is greatly decreased when pure lutein is orally consumed (Sant'Anna, V., Gurak, Marczak, \& Tessaro, 2013; Ye, Lei, Wang, \& Zhao, 2017). Solid dispersions have demonstrated the ability to improve the bioavailability and water affinity of food-related bioactive substances such as curcumin and betacarotene (Frizon, Oliveira, Maria, Lina, \& Maldonado, 2013; Gangurde et al., 2015; Gul et al., 2015). It has been successfully applied to non-food drugs like felodipine (Karavas, Ktistis, Xenakis, \& Georgarakis, 2006), loratadine (Frizon et al., 2013), and carbamazepine (Sethia \& Squillante, 2004) demonstrating the versatility of this approach. Recently, lutein was encapsulated by the solid dispersion method (Silva, Geiss, et al., 2017, Silva, Silva, et al., 2017) yielding nanoparticles with uniform sizes and considerable gains in water affinity. Encapsulated lutein improved the memory of mice without causing inflammatory damage per se, which is valuable information due to concerns with the safety of adding nanomaterials to foodstuff.

There has been a search for natural compounds that could act as acetylcholinesterase (AChE) inhibitors mainly because it is believed that the damage to the cholinergic system is closely related to brain dysfunctions such as affective disorders, depression, schizophrenia and

E-mail address: odinei@utfpr.edu.br (O. Hess Gonçalves). 
delirium (Contestabile, 2011), memory loss in ageing (Gallagher \& Colombo, 1995), and Parkinson's disease (Ventura et al., 2010). In the case of Alzheimer's disease (AD), the Cholinergic Hypothesis is the most accepted theory (Järvinen, Vuorela, Hatakka, \& Fallarero, 2011; Motta et al., 2016; Vandeput et al., 2015), stating that the loss of cholinergic function contributes to the decrease in cognitive activity associated with AD (Terry \& Buccafusco, 2003; Viegas Junior, Bolzani, Furlan, Fraga, \& Barreiro, 2004). The cholinergic hypothesis of learning has driven drug development for the treatment of $\mathrm{AD}$ and other types of dementia. Cholinergic inhibitors have presented promising results (Contestabile, 2011; Hornick et al., 2008; Motta et al., 2016), and drugs such as tacrine, donepezil, rivastigmine, and galantamine were approved by the United States Food and Drug Administration (FDA) as AChE inhibitors. The substances have been showing modest improvements in patients with early stages of $\mathrm{AD}$, and there is a need for effective drugs with fast penetration into the central nervous system, long duration, and low toxicity that act as AChE inhibitors (Contestabile, 2011; Hornick et al., 2008; Motta et al., 2016).

Although the efficacy of free lutein to inhibit the action of AChE is well-studied, the effects of nanoencapsulation are still to be determined. Also, there is a need to investigate if encapsulated lutein would inhibit the enzyme in aqueous media (without good lutein solvents such as ethanol), which could represent an improvement in nutraceutical food development. The objective of this study was to assess the action of lutein-loaded nanoparticles on the acetylcholinesterase enzyme using in vitro experiments and the interaction with the enzyme using in silico docking simulations.

\section{Materials and methods}

\subsection{Material}

Lutein ( $90 \%$ purity, kindly donated by Pincredit Bio-tech Co.), polyvinylpyrrolidone (PVP, 40,000 $\mathrm{g} \cdot \mathrm{mol}^{-1}$, Sigma-Aldrich), Tween 80 (Dinâmica), and ethanol (99.5\%, Neon) were used to prepare the nanoparticles. It is worth noting that both PVP and Tween 80 are considered as food safe additives. Ethanol was also used to prepare the free lutein solutions for the in vitro tests. The reagents used for the acetylcholinesterase (AChE) activity were 5',5-dithiobis (2-nitrobenzoic acid) (DTNB, 98\%, Sigma-Aldrich), potassium phosphate buffer (TFK) with a pH of 7.5 (99.9\%, Neon), and acetylthiocholine (ASCh) (SigmaAldrich, 99\%). The enzyme was obtained from the homogenization of rat brains in tris(hydroxymethyl)aminomethane hydrochloride (Tris$\mathrm{HCl}, 50 \mathrm{Mm}, \mathrm{pH} 7.4,99 \%$, Dinâmica) at 1:10 (brain:Tris- $\mathrm{HCl}$ mass proportion) that was kindly donated by the Federal University of Santa Maria under the Ethics Committee Protocol no. 2729/2014-GRE.

\subsection{Lutein-loaded nanoparticle production and characterization}

Nanoparticles were produced using the methodology described in detail bySilva, Geiss, et al. (2017) and Silva, Silva, et al. (2017). Briefly, PVP $(0.360 \mathrm{~g})$ was dissolved in ethanol $(22.5 \mathrm{~mL})$ under gentle stirring for 1 minute. After, Tween $80(0.045 \mathrm{~g})$ and lutein $(0.045 \mathrm{~g})$ were added and mixed for another minute. The solution was then sonicated (Fisher Scientific, $120 \mathrm{~W}, 1 / 8^{\prime}$ probe) for 5 minutes under a pulsed regime (30 seconds on and 10 seconds off) in an ice bath. Solvent was then evaporated in an air circulation oven at $40^{\circ} \mathrm{C}$ for $24 \mathrm{~h}$ protected from light.

The thermal properties of the nanoparticles were investigated by Differential Scanning Calorimetry (DSC, Perkin Elmer 4000). Samples were accommodated in sealed aluminum pans under a nitrogen flow $\left(50 \mathrm{~mL} \cdot \mathrm{min}^{-1}\right.$ ) and heated from 20 to $360^{\circ} \mathrm{C}$ at $20^{\circ} \mathrm{C} \cdot \mathrm{min}^{-1}$. Fourier Transform Infrared (FTIR) spectra were acquired using a Frontier Perkin Elmer equipment in potassium bromide pellets, with a resolution of $2 \mathrm{~cm}^{-1}$ from 4000 to $400 \mathrm{~cm}^{-1}$ with 32 cumulative scans (peaks were normalized to allow comparison). For DSC and FTIR experiments, all samples were simultaneously stored in a vessel protected from light
( $25^{\circ} \mathrm{C}$ and $60 \%$ relative humidity) for 7 days before analyses in order to avoid the influence of adsorbed air moisture. Physical mixtures of lutein and PVP were obtained by manually mixing them in a glass vessel in order to qualitatively highlight the differences between actual encapsulation and simple mixture. X-ray diffraction analyses (XRD, Bruker, D8 Advance) were carried out from $3^{\circ}$ to $60^{\circ}(2 \theta)$ at $5.9^{\circ} \cdot \mathrm{min}^{-1}$ using $\mathrm{Cu} \mathrm{Ka}$ radiation generated at $40 \mathrm{KV}$ and $35 \mathrm{~mA}$. Transmission Electron Microscopy (TEM; JEOL model JEM 2100, $200 \mathrm{kV}$ ) was used to evaluate the nanoparticles morphology. Diluted samples were dripped onto 300 mesh parlodium-covered copper grids. The interaction intensity (as defined by Karavas et al. (2006)) between PVP and lutein was investigated by UV-Vis spectroscopy (Ocean Optics, model USB650-UV-VIS Red Tide) at $446 \mathrm{~nm}$. Specified amounts of PVP were added to a lutein ethanolic solution $\left(0.1 \mathrm{mg} \cdot \mathrm{mL}^{-1}\right)$ and the absorbance was recorded. The interaction intensity (F) was calculated by Eq. (1), where $\mathrm{A}$ is the absorbance of the PVP-lutein ethanolic solution and $\mathrm{A}_{0}$ is the absorbance of the lutein ethanolic solution.

$F(\%)=100 \frac{A-A_{0}}{A_{0}}$

\subsection{AChE activity assay}

Adult rats were anesthetized and sacrificed, then their brains were removed and homogenized in Tris- $\mathrm{HCl}, 50 \mathrm{Mm}, \mathrm{pH} 7.4,1: 10$ (wt/vol). The homogenized solution was centrifuged at $10,000 \mathrm{rpm}$ for $10 \mathrm{~min}-$ utes, and the supernatant (S1) was used for enzymatic assays. Three different systems were prepared to evaluate the influence of lutein on the activity of acetylcholinesterase (AChE): (1) water dispersion of lutein-loaded nanoparticles; (2) water dispersion of lutein; and (3) lutein in ethanol (at the concentration used, ethanol did not inhibit AChE activity per se). All systems were prepared in the following concentrations: 100,200 , and $300 \mu \mathrm{M}$. Also, the water dispersion of PVP was tested at the same higher lutein concentration $(300 \mu \mathrm{M})$ to verify the per $s e$ effect of PVP. Free lutein was evaluated in ethanol because it was not possible to dissolve it in water as expected due to its high hydrophobic nature. Nanoparticles were dispersed in water to determine if this compound would present activity even in the presence of a poor lutein solvent (water).

The colorimetric methodology used was previously described by Ellman, Courtney, Andres, and Featherstone (1961) and modified by Pereira, Adams, and Silva (2004). The assay medium contained a final concentration of $1.05 \mathrm{mM}$ DTNB - 5',5-dithiobis (2-nitrobenzoic acid), $24 \mathrm{mM}$ potassium phosphate buffer $(\mathrm{pH}=7.5)$, and $75 \mu \mathrm{L}$ of supernatant solution (S1). The product of the reaction was determined by adding $0.84 \mathrm{mM}$ of acetyltiocholine (ASCh), and the reaction rate was measured by the increase in absorbance at $412 \mathrm{~nm}$ for 3 minutes every 30 seconds using a UV-Vis spectrophotometer (OceanOptics model USB650). The inhibition rate was determined using Eq. (2), where 13.6 is the molar extinction coefficient, 0.075 is the volume of supernatant solution (S1), $\Delta$ absorbance is the absorbance variation per minute.

Reaction rate $\left(\frac{m o l}{L . \mathrm{h}}\right)=\frac{\Delta \text { absorbance }}{13.6 \hat{\mathrm{A}} \cdot 0.075}$

\subsection{Reaction kinetics}

Kinetics parameter determination was carried out using four acetylthiocholine concentrations $(0.8,0.4,0.2,0.1$, and $0 \mathrm{mM})$ and two concentrations of lutein (200 and $300 \mu \mathrm{M})$, as follows: (1) water dispersion of lutein-loaded nanoparticles; (2) water dispersion of lutein; and (3) lutein in ethanol. The inhibition constant of the enzyme-substrate-inhibitor complex $\left(\mathrm{K}_{\mathrm{i}}\right)$ was obtained through the LineweaverBurk methodology using Prism GraphPad 5.0 software. 


\subsection{Docking simulations using AutoDock Vina}

Lutein 2D structure preparation and 3D structure optimization were performed using Marvin Sketch 16.4 software (www.chemaxon.com). AutoDockTools 1.5.2 (ADT) was then used to convert the 3D structure of lutein to the PDBQT file format required for docking. ADT performs several preparation steps including merging non-polar hydrogens, adding Gasteiger charges, and setting up rotatable bonds. The AChE Xray crystal structure (PDB: 5FKJ) used was obtained from the Protein Data Bank (PDB) (http://www.rcsb.org). The co-crystallized ligand was extracted from the PDB file and ADT was used to assign polar hydrogens and Gasteiger charges, as well as save the AChE structure in the required PDBQT file format. Autodock Vina was then used to perform molecular docking in a selected grid area that encompassed the complete binding site, presenting the following parameters: center $\mathrm{X}, \mathrm{Y}$, and $\mathrm{Z}$ coordinates of $15.5,-20$, and 93.2, respectively; grid size XYZ dimensions of 30 by 30 by $30 \AA$; and an exhaustiveness of 16 (Hashmi \& Al-Salam, 2015). The conformational cluster analysis based on estimated free energy calculations was carried out for screening the best ligand-protein binding complex. Docking simulations were performed in an AMD Opteron 6128 processor computer. All figures with structure representations were prepared using PyMOL (The PyMOL Molecular Graphics System, Version 1.3, Schrödinger, LLC).

\subsection{Statistical analyses}

The interaction intensity $(\mathrm{F})$ procedure was carried out in triplicate and results are presented as mean \pm standard deviation. One-way ANOVA was performed to investigate significant differences among treatments in the in vitro experiments. Tukey's post hoc test was selected to identify the pairs of means that differ from each other. Analyses were conducted in duplicate (4-6 repetitions per group) using GraphPad Prism 5 software with a 5\% significance level.

\section{Results and discussion}

\subsection{Nanoparticles characterization}

Fig. 1 presents images of lutein-loaded nanoparticles in water, free lutein in water, and free lutein dissolved in ethanol. Morphological (Transmission Electron Microscopy) and physicochemical (X-ray diffraction, FTIR spectra and DSC thermograms are presented in Fig. 2. Figs. S1 and S2 (Supplementary Material) present the FTIR and DSC results, respectively, of physical mixtures of PVP and lutein in different proportions. Table S1 (Supplementary Material) presents the enthalpy involved in the water loss for PVP, lutein-loaded nanoparticles, and the lutein-PVP physical mixture. Enthalpies were calculated based on the mass proportion of lutein and PVP in the nanoparticles and the physical mixture (10\%wt lutein concentration).

Nanoparticles with diameters from 50 to $200 \mathrm{\eta m}$ were observed in the microscopy images (Fig. 2a) presenting fairly spherical morphology. When dispersed in water, the nanoparticles formed a colloidally stable dispersion, as may be seen in Fig. 1a, which is very similar to the lutein ethanolic solution in Fig. 1c (it is worth noting that lutein and PVP are highly soluble in ethanol). On the other hand, when dispersed in water, lutein formed coarse agglomerates which were prone to precipitate.

The Differential Scanning Calorimetry of lutein showed an exothermic peak at $112{ }^{\circ} \mathrm{C}$ which is related to the degradation of its structure (Miguel, Martín, Mattea, \& Cocero, 2008). An endothermic peak around $66^{\circ} \mathrm{C}$ was detected for free lutein (Fig. 2d). This peak was also visible in the physical mixture but absent in the lutein-loaded nanoparticles. This fact is indicative of the efficient encapsulation of compounds inside the encapsulation matrix (Dandekar, Jain, Patil, Dhumal, \& Tiwari, 2010; Mohan, Gupta, Jaggi, \& Chauhan, 2010; da Silva-Buzanello et al., 2016; Yallapu, Jaggi, \& Chauhan, 2010). The PVP thermogram showed a large endothermic peak caused by water loss (Paradkar, Ambike, Jadhav, \& Mahadik, 2004) with an associated enthalpy of $314 \mathrm{~J} \cdot \mathrm{g}^{-1}$. The same peak is visible in the nanoparticles and the physical mixture samples, as expected. In the case of the physical mixture, the enthalpy is slightly higher probably because of the endothermic peak associated with free lutein around $66^{\circ} \mathrm{C}$ (as indicated in Fig. 2d). The enthalpy of evaporation was found to decrease in the case of the nanoparticles sample but not in the physical mixture, even considering that all samples present the same mass proportion of PVP and lutein. This may be caused by the interaction through hydrogen binding between carbonyl from PVP and hydroxyl from lutein in the nanoparticles. Previous works (Silva, Geiss, et al., 2017, Silva, Silva, et al., 2017) have reported a decrease in the evaporation enthalpy for nanoparticles with increasing lutein concentrations.

The XRD analysis of lutein showed two peaks at approximately 20 and $24^{\circ}$ which are characteristic of crystalline structures (Fig. 2b). The PVP diffractogram showed no peaks, which is typical of amorphous materials. The same is valid for the nanoparticles and the physical mixture, probably due to the low concentration of lutein in the sample. The FTIR spectra of pure lutein presented its characteristic groups at 2850 and $2920 \mathrm{~cm}^{-1}\left(\mathrm{CH}_{2}\right.$ and $\mathrm{CH}_{3}$ stretching vibrations), $1044 \mathrm{~cm}^{-1}$

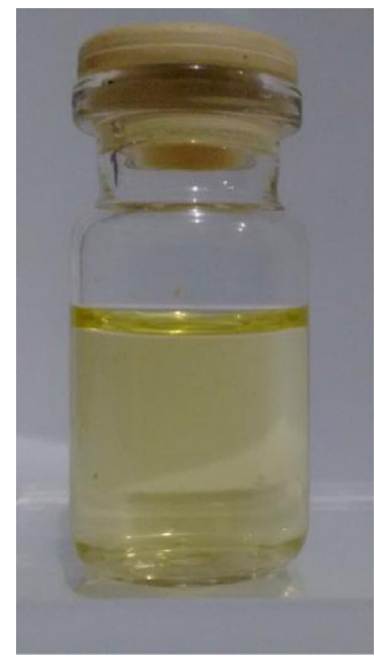

(a)

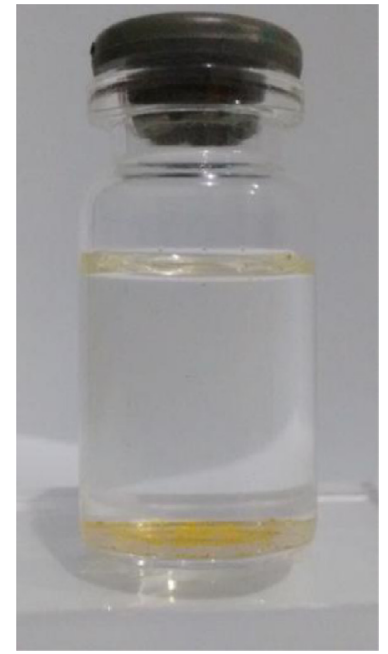

(b)

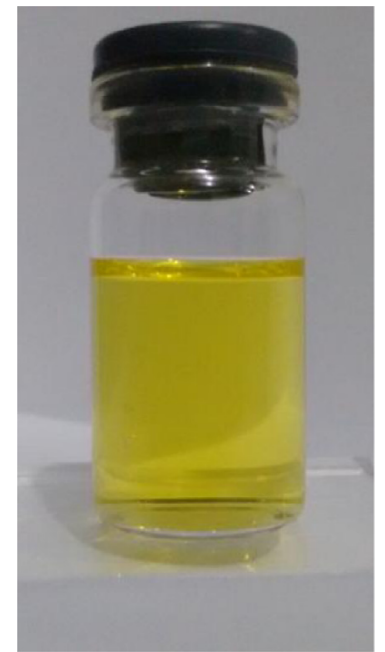

(c)

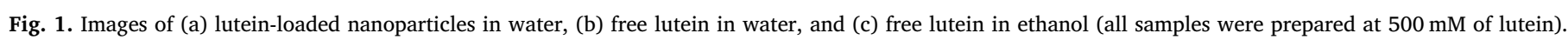




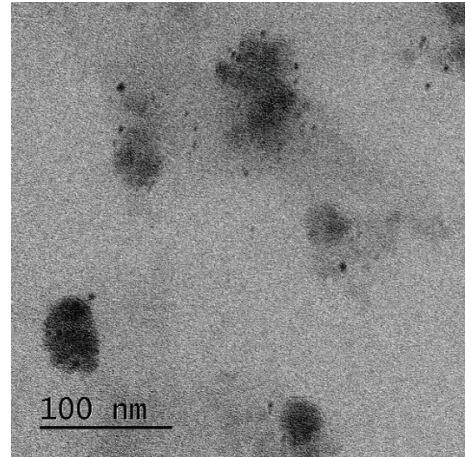

(a)

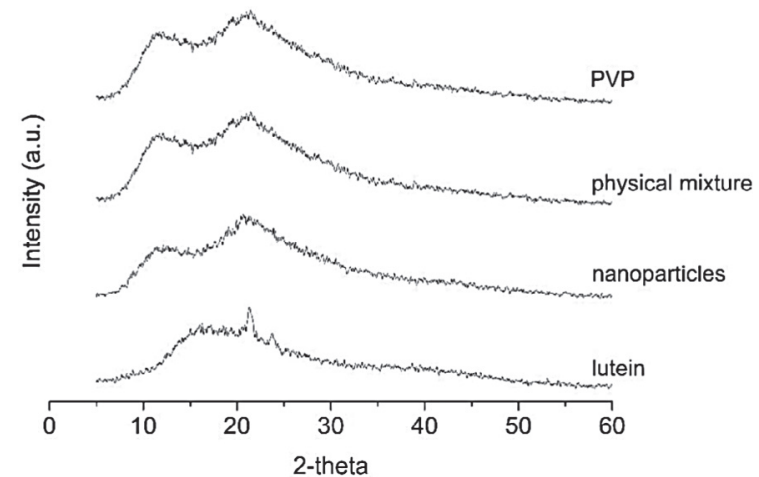

(b)

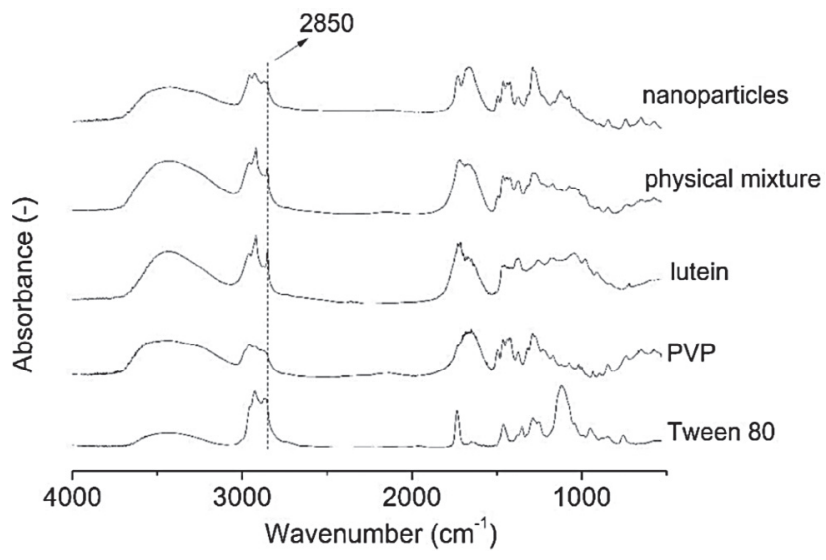

(c)
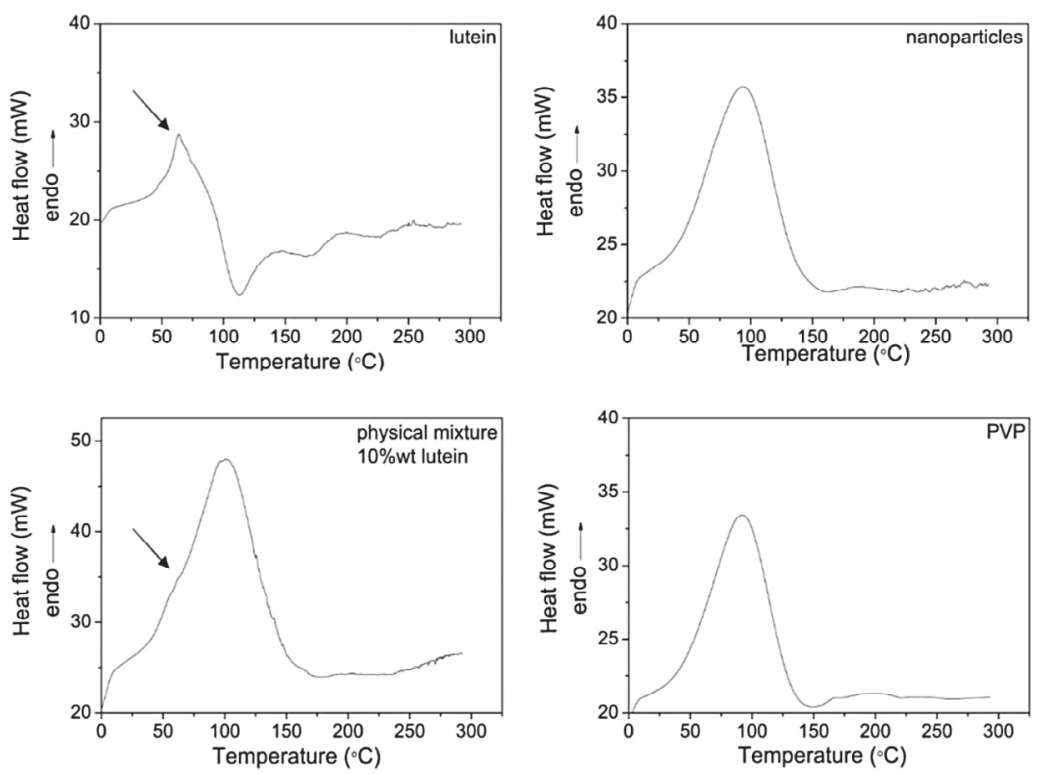

(d)

Fig. 2. Characterization results of the lutein-loaded nanoparticles.

(CH group), $1371 \mathrm{~cm}^{-1}$ (dimethyl group) and $3435 \mathrm{~cm}^{-1}$ (hydroxyl group). The $\mathrm{C}-\mathrm{H}$ out of the plane bending vibration was present in lutein at 979 and $833 \mathrm{~cm}^{-1}$ (Ranganathan, Hindupur, \& Vallikannan, 2016; Silva, Geiss, et al., 2017, Silva, Silva, et al., 2017). Although many absorption bands of lutein overlapped with those of PVP and Tween 80 , the absorption band at $2850 \mathrm{~cm}^{-1}$ is present in lutein and physical mixture spectra, while it was attenuated in the nanoparticles. This is indicative that lutein is embedded inside the nanoparticles.
FTIR spectra, DSC thermograms, TEM images and the X-Ray diffraction patterns together represent a strong indicative that encapsulation was carried out successfully.

Fig. 3 presents the interaction intensity factor $(F)$ between lutein and PVP for different mass proportions.

The interaction assay was made using ethanol, which is the same solvent used to obtain the nanoparticles. This means that results indicate the behavior of PVP and lutein during the encapsulating process 


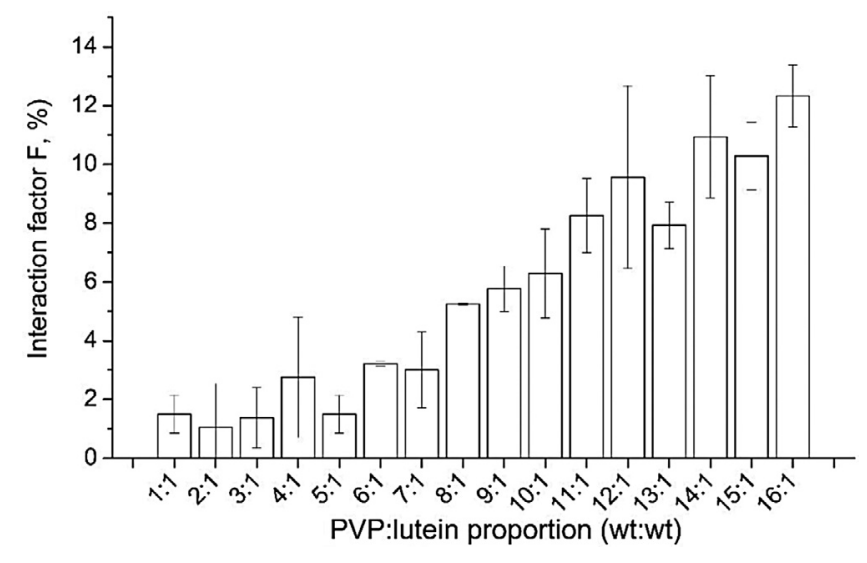

Fig. 3. Interaction intensity factor (F) between PVP and lutein.

(Silva et al., 2017). It is possible to observe that there was an increase in the interaction intensity when decreasing the lutein concentration. Although a steady increase in the interaction was observed in the range of lutein:PVP ratio, the proportion 8:1 was chosen because it represented an increase in the interaction factor when compared to lower ratio studied. One possible mechanism for the formation of the particles was suggested by Zhao and coauthors (Zhao, Cheng, Jiang, Yao, \& Han, 2014) involving the formation of hydrogen bonds between lutein hydroxyl groups and PVP carbonyl groups. The formation of such bonds involving carbonyl groups from the same PVP chain could lead to a crosslinked structure which may precipitate from the solvent forming the nanoparticles with the aid of the sonication device. The system is further stabilized by the presence of Tween 80 , which is a strong and high HLB (hydrophilic-lipophilic balance) emulsifier.

\subsection{AChE activity assay and reaction kinetics}

Fig. 4 presents the AChE activity in rat brains for free and encapsulated lutein. Fig. 5 presents the Michaelis-Menten substrate competition assays for encapsulated and pure lutein.

The AChE activity was significantly inhibited at 200 and $300 \mu \mathrm{M}$ for both free lutein and lutein-loaded nanoparticles. For $200 \mu \mathrm{M}$, the activity differed for each, as demonstrated by Tukey's test. It is worth noting that lutein-loaded nanoparticles presented inhibition even when dispersed in water, without the aid of any other solvent such as ethanol. Since lutein is poorly soluble in water, this indicates that the encapsulation was able to improve the water affinity of lutein. At $300 \mu \mathrm{M}$,

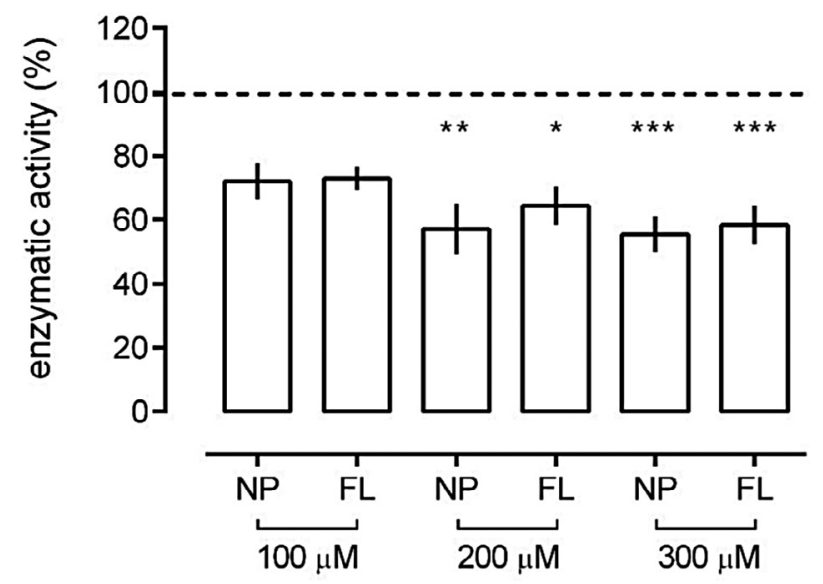

Fig. 4. In vitro AChE activity in rat brain tissue. (NP: lutein-loaded nanoparticles; FL: free lutein). $n=4-6$ animals per group, mean \pm SD.; One-way analyses of variance (ANOVA) followed by Tukey's test; *p $<0.05$, $* * \mathrm{p}<0.01, * * * \mathrm{p}<0.001$ in comparison to the control group.
AChE activity was statistically the same for free lutein and nanoparticles ( $\mathrm{p}<0.001$ when compared to the control group). PVP per se did not alter AChE activity (data not shown in Fig. 5).

Regarding nanoparticles, Silva, Geiss, et al., 2017, Silva, Silva, et al., 2017 have applied lutein nanoparticles for enhancing the memory of mice and verified that 1.5 and $10 \mathrm{mg} \cdot \mathrm{kg}^{-1}$ were able to cause this effect. As AChE is related to memory activity and cognition, the effect of increased memory of the mice may be related to the activity of AChE during the longer actuation time of ACh, which is a neurotransmitter and substrate of AChE.

The Michaelis-Menten assays demonstrated that free lutein and lutein-loaded nanoparticles were able to decrease the AChE activity when compared to the control $\left(\mathrm{H}_{2} \mathrm{O}\right.$ curve). The Lineweaver-Burk methodology indicated that both samples presented inhibition of competitive mixed-type, with $\mathrm{K}_{\mathrm{i}}$ values of $265 \mu \mathrm{M}$ and $262 \mu \mathrm{M}$, respectively. López, Campoy, Pascual-Villalobos, Muñoz-Delgado, and Vidal (2015) studied the inhibition of AChE activity of the electric eel by monoterpenoids and phenylpropanoids, finding that these compounds may be considered weaker inhibitors than known alkaloids such as galanthamine. The essential oil of Salvia lavandulaefolia and its main constituents ( $\alpha$ pinene, 1,8-cineole, and camphor) were found to be uncompetitive reversible inhibitors for the activity of human erythrocyte AChE.

\subsection{In silico docking}

In silico docking was implemented in order to understand the possible mechanism of action of to evaluate the possible interactions between lutein and on the enzyme AChE. Fig. 6A shows a detailed analysis of the predicted binding conformation of lutein, obtained using AutoDock Vina docking software, while Fig. 6B presents the complete AChE surface representation, highlighting the binding pocket.

The X-ray structure of AChE (PDB:5FKJ) selected for this docking study presents C-547, an alkyl ammonium derivative that is a known AChE inhibitor and which was co-crystallized with the AChE structure, being also presented for comparison in Fig. 6 (Kharlamova et al., 2016). This AChE structure was selected due to overall C-547 structural resemblance with lutein. One of the terminal hydroxyl group is predicted to form a hydrogen bond with GLU202, deep inside the AChE binding pocket, while the other terminal hydroxyl group is projected towards the exterior while forming a second hydrogen bond with ARG276. The lutein hydrophobic skeleton is predicted to occupy the elongated AChE binding site in a similar fashion to C-547, stabilized by the formation of hydrophobic interactions with several hydrophobic residues (Fig. 6A). This structural analysis suggests the delivery of lutein to AChE as probably the critical step of lutein action as an AChE inhibitor, due to his hydrophobic nature. Once lutein is delivered it is readily accommodated by the also largely hydrophobic AChE binding site. A $\mathrm{K}_{\mathrm{i}}$ value of $24 \mathrm{nM}$ for lutein AChE inhibition was also predicted by AutoDock Vina software. This value differs from the experimental value found above which could be explained by the fact that the docking simulation implemented here does not take into account the chemical environment in which the reaction takes place thus neglecting the possible interference of other molecules and the difficulty of lutein to reach AChE binding pocket due to his hydrophobicity. Another fact is that reaction kinetics could also influence on the experimental value of $\mathrm{K}_{\mathrm{i}}$. Nonetheless, both experimental and docking results agreed that lutein can act as an inhibitor of the AChE activity. Overall, a satisfactory mechanism prediction of AChE inhibition by lutein was predicted.

\section{Conclusion}

Lutein, a bioactive natural substance, was nanoencapsulated using the solid dispersion method. The nanoparticles were evaluated in an aqueous medium to verify if the water affinity of lutein was affected by the encapsulation. In summary, this study demonstrated that the encapsulation of lutein using the solid dispersion technique was efficient 


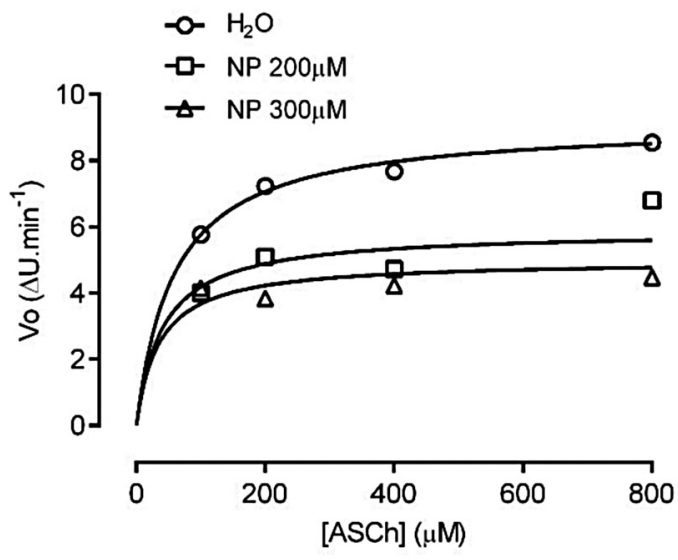

(a)

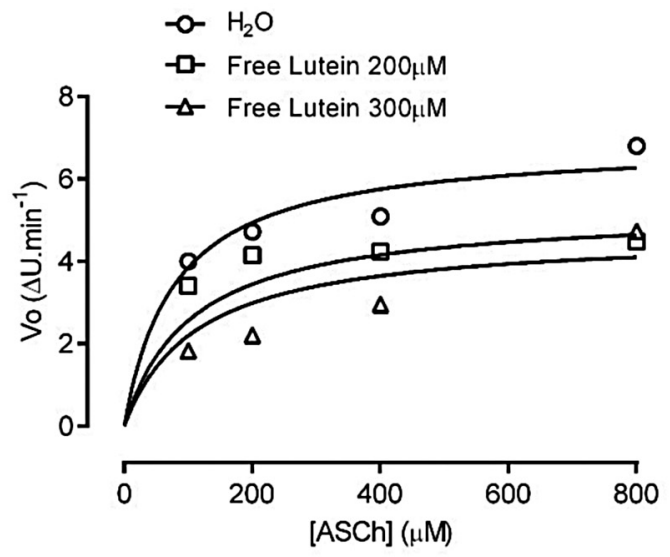

(b)

Fig. 5. Michaelis-Menten substrate competition assays of (a) encapsulated lutein and (b) pure lutein, both for rat brain AChE activity.

since colloidal stability was improved. The physicochemical characterization showed the formation of hydrogen bonds with PVP, leading to the development of spherical nanometric particles. The in vitro analyses using rat brain tissue demonstrated that both lutein and luteinloaded nanoparticles inhibited AChE activity in both forms. It is important to point out that there was no significant loss in activity for the encapsulated lutein even when the assay was conducted in an aqueous medium. The results of the kinetic study showed that both free and encapsulated lutein had a mixed-type inhibition of the AChE activity. In silico docking showed the anchorage of lutein to the AChE structure, causing an inhibition of its activity and corroborating the in vitro results. Also, the AChE-lutein complex is not reported in the Protein Data Bank (PDB), meaning that these results contribute to the understanding of the action mechanism of lutein.

\section{Conflicts of Interest}

Authors have no conflicts to declare.

\section{Declaration of Competing Interest}

The authors declare that they have no known competing financial interests or personal relationships that could have appeared to influence the work reported in this paper.

\section{Acknowledgements}

Authors thank CAPES, CNPq and Fundação Araucária for the support.

\section{Appendix A. Supplementary data}

Supplementary data to this article can be found online at https:// doi.org/10.1016/j.foodchem.2019.125523.

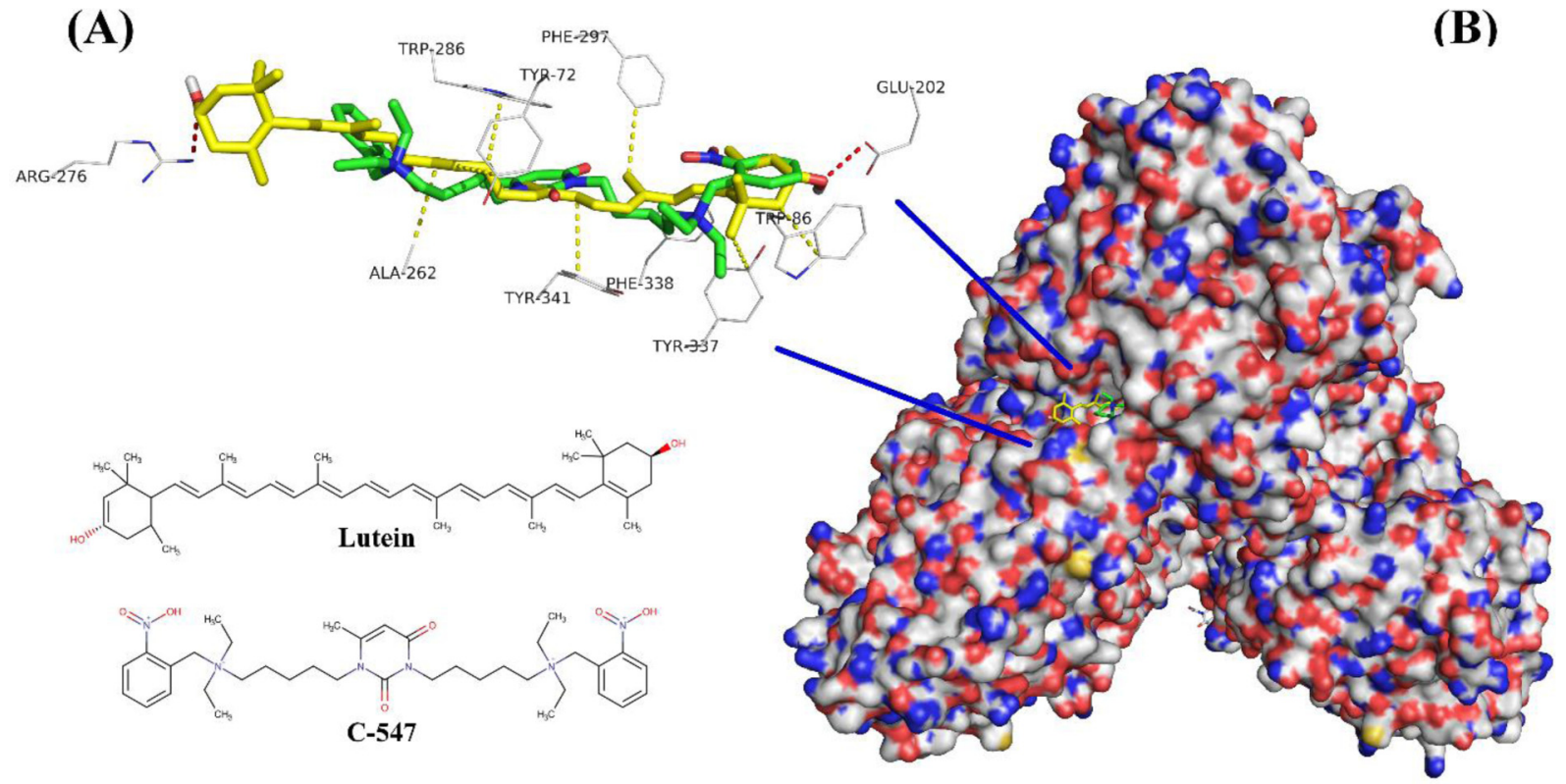

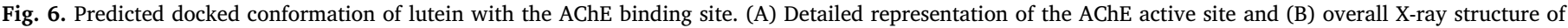

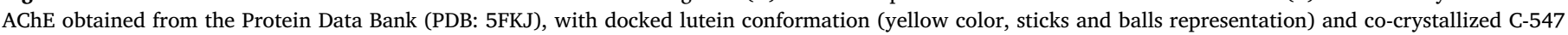

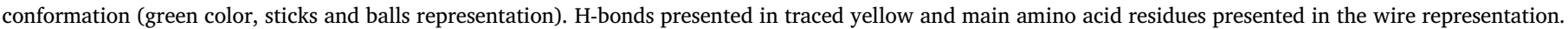
(For interpretation of the references to colour in this figure legend, the reader is referred to the web version of this article.) 


\section{References}

Alves-Rodrigues, A., \& Shao, A. (2004). The science behind lutein. Toxicology Letters, 150(1), 57-83. https://doi.org/10.1016/j.toxlet.2003.10.031.

Contestabile, A. (2011). The history of the cholinergic hypothesis. Behavioural Brain Research, 221(2), 334-340. https://doi.org/10.1016/j.bbr.2009.12.044.

Dandekar, P. P., Jain, R., Patil, S., Dhumal, R., \& Tiwari, D. (2010). Curcumin-loaded hydrogel nanoparticles: Application in anti-malarial therapy and toxicological evaluation. Journal of Pharmaceutical Sciences, 99(12), 4992-5010. https://doi.org/10. 1002/jps.

Ellman, G. L., Courtney, K. D., Andres, V., \& Featherstone, R. M. (1961). A new and rapid colorimetric determination of acetylcholinesterase activity. Biochemical Pharmacology, 7(2), 88-95. https://doi.org/10.1016/0006-2952(61)90145-9.

Frizon, F., Oliveira, J. De, Maria, C., Lina, M., \& Maldonado, J. (2013). Dissolution rate enhancement of loratadine in polyvinylpyrrolidone K-30 solid dispersions by solvent methods. Powder Technology, 235, 532-539.

Gallagher, M., \& Colombo, P. J. (1995). Ageing: The cholinergic hypothesis of cognitive decline. Current Opinion in Neurobiology.

Gangurde, A. B., Kundaikar, H. S., Javeer, S. D., Jaiswar, D. R., Degani, M. S., \& Amin, P. D. (2015). Enhanced solubility and dissolution of curcumin by a hydrophilic polymer solid dispersion and its insilico molecular modeling studies. Journal of Drug Delivery Science and Technology, 29, 226-237. https://doi.org/10.1016/j.jddst.2015.08.005.

Gul, K., Tak, A., Singh, A. K., Singh, P., Yousuf, B., \& Wani, A. A. (2015). Chemistry, encapsulation, and health benefits of beta-carotene - A review. Cogent Food \& Agriculture, 1(1), 1-12. https://doi.org/10.1080/23311932.2015.1018696.

Hashmi, S., \& Al-Salam, S. (2015). Acute myocardial infarction and myocardial ischemiareperfusion injury: A comparison. International Journal of Clinical and Experimental Pathology, 8(8), 8786-8796. https://doi.org/10.1002/jcc.

Hornick, A., Schwaiger, S., Rollinger, J. M., Vo, N. P., Prast, H., \& Stuppner, H. (2008) Extracts and constituents of Leontopodium alpinum enhance cholinergic transmission: Brain ACh increasing and memory improving properties. Biochemical Pharmacology, 76(2), 236-248. https://doi.org/10.1016/j.bcp.2008.04.015.

Järvinen, P., Vuorela, P., Hatakka, A., \& Fallarero, A. (2011). Potency determinations of acetylcholinesterase inhibitors using Ellman's reaction-based assay in screening: Effect of assay variants. Anal Biochem. 408, 166-168. https://doi.org/10.1016/j.ab. 2010.09.018.

Karavas, E., Ktistis, G., Xenakis, A., \& Georgarakis, E. (2006). Effect of hydrogen bonding interactions on the release mechanism of felodipine from nanodispersions with polyvinylpyrrolidone. European Journal of Pharmaceutics and Biopharmaceutics, 63(2), 103-114. https://doi.org/10.1016/j.ejpb.2006.01.016.

Kharlamova, A. D., Lushchekina, S. V., Petrov, K. A., Kots, E. D., Nachon, F., Villardwandhammer, M., ... Masson, P. (2016). Slow-binding inhibition of acetylcholinesterase by an alkylammonium derivative of 6-methyluracil: Mechanism and possible advantages for myasthenia gravis treatment. Biochemical Journal, 473, 1225-1236. https://doi.org/10.1042/BCJ20160084.

Kim, J. E., Clark, R. M., Park, Y., Lee, J., \& Fernandez, M. L. (2012). Lutein decreases oxidative stress and inflammation in liver and eyes of guinea pigs fed a hypercholesterolemic diet. Nutr. Res. Pract. 6(2), 113-119.

Lakshminarayana, R., Aruna, G., Sangeetha, R. K., Bhaskar, N., Divakar, S., \& Baskaran, V. (2008). Possible degradation/biotransformation of lutein in vitro and in vivo: Isolation and structural elucidation of lutein metabolites by HPLC and LC-MS (atmospheric pressure chemical ionization). Free Radical Biology and Medicine, 45(7), 982-993. https://doi.org/10.1016/j.freeradbiomed.2008.06.011.

Li, R., Turner, S. D., \& Brautigan, D. L. (2015). Xanthophylls lutein and zeaxanthin modify gene expression and induce synthesis of hyaluronan in keratinocyte model of human skin. Biochemistry and Biophysics Reports, 4, 52-58. https://doi.org/10.1016/j.bbrep. 2015.08.012.

López, M. D., Campoy, F. J., Pascual-Villalobos, M. J., Muñoz-Delgado, E., \& Vidal, C. J. (2015). Acetylcholinesterase activity of electric eel is increased or decreased by selected monoterpenoids and phenylpropanoids in a concentration-dependent manner. Chemico-Biological Interactions, 229, 36-43. https://doi.org/10.1016/j.cbi.2015.01. 006.

Miguel, F., Martín, A., Mattea, F., \& Cocero, M. J. (2008). Precipitation of lutein and coprecipitation of lutein and poly-lactic acid with the supercritical anti-solvent process. Chemical Engineering and Processing: Process Intensification, 47(9-10), 1594-1602. https://doi.org/10.1016/j.cep.2007.07.008.

Mohan, M., Gupta, B. K., Jaggi, M., \& Chauhan, S. C. (2010). Journal of colloid and interface science fabrication of curcumin encapsulated PLGA nanoparticles for improved therapeutic effects in metastatic cancer cells. Journal of Colloid And Interface Science, 351(1), 19-29. https://doi.org/10.1016/j.jcis.2010.05.022.

Motta, F., Silva, R., Castro, G. De, Boni, S., Jorge, M., Guimar, R., ... Romeiro, S. (2016) Chemistry Cardanol-derived AChE inhibitors : Towards the development of dual binding derivatives for Alzheimer's disease Laís Fl a be. Europen Journal of Medicinal, 108, 687-700. https://doi.org/10.1016/j.ejmech.2015.12.024.

Paradkar, A., Ambike, A. A., Jadhav, B. K., \& Mahadik, K. R. (2004). Characterization of curcumin - PVP solid dispersion obtained by spray drying. International Journal of Pharmaceutics, 271, 281-286. https://doi.org/10.1016/j.ijpharm.2003.11.014.

Pereira, M. E., Adams, A. I. H., \& Silva, N. S. (2004). Hexanedione inhibits rat brain acetylcholinesterase activity in vitro. Toxicology Letters, 146(5), 269-274. https://doi. org/10.1016/j.toxlet.2003.10.009.

Ranganathan, A., Hindupur, R., \& Vallikannan, B. (2016). Biocompatible lutein-polymerlipid nanocapsules: Acute and subacute toxicity and bioavailability in mice. Materials Science and Engineering C, 69, 1318-1327. https://doi.org/10.1016/j.msec.2016.08. 029.

Sant'Anna, V., Gurak, P. D., Marczak, L. F. D., \& Tessaro, I. C. (2013). Tracking bioactive compounds with colour changes in foods - A review. Dyes and Pigments, 98(3), 601-608. https://doi.org/10.1016/j.dyepig.2013.04.011.

Sethia, S., \& Squillante, E. (2004). Solid dispersion of carbamazepine in PVP K30 by conventional solvent evaporation and supercritical methods. International Journal of Pharmaceutics, 272(1-2), 1-10. https://doi.org/10.1016/j.ijpharm.2003.11.025.

da Silva-Buzanello, R. A., de Souza, M. F., de Oliveira, D. A., Bona, E., Leimann, F. V., Cardozo Filho, L., ... Gonçalves, O. H. (2016). Preparation of curcumin-loaded nanoparticles and determination of the antioxidant potential of curcumin after encapsulation. Polímeros, 26(3), 207-214. https://doi.org/10.1590/0104-1428.2246.

Silva, J. T. do P., Geiss, J. M. T., Oliveira Brum, S. M. E. da S., Sagae, S. C., Becker, D., ... Gonçalves, O. H. (2017). Nanoencapsulation of lutein and its effect on mice's declarative memory. Materials Science and Engineering C, 76, 1005-1011. https://doi. org/10.1016/j.msec.2017.03.212.

Silva, J. T. P., Silva, A. C., Geiss, J. M. T., Araújo, P. H. H., Becker, D., Bracht, L., ... Gonçalves, H. O. (2017). Analytical validation of an ultraviolet - visible procedure for determining lutein concentration and application to lutein-loaded nanoparticles. Food Chemistry, 230, 336-342. https://doi.org/10.1016/j.foodchem.2017.03.059.

Terry, A. V., Jr, \& Buccafusco, J. J. (2003). The cholinergic hypothesis of age and Alzheimer's Disease - Related cognitive deficits: recent challenges and their implications for novel drug development. The Journal of Pharmacology and Experimental Therapeutics, 306(3), 821-827. https://doi.org/10.1124/jpet.102.041616.For.

Vandeput, M., Parsajoo, C., Vanheuverzwijn, J., Patris, S., Yardim, Y., Sarakbi, A., ... Kauffmann, J. (2015). Journal of pharmaceutical and biomedical analysis flowthrough enzyme immobilized amperometric detector for the rapid screening of acetylcholinesterase inhibitors by flow injection analysis. Journal of Pharmaceutical and Biomedical Analysis, 102, 267-275. https://doi.org/10.1016/j.jpba.2014.09.012.

Ventura, A. L. M., Abreu, P. A., Freitas, R. C. C., Sathler, P. C., Loureiro, N., \& Castro, H. C. (2010). Sistema colinérgico: Revisitando receptores, regulação e a relação com a doença de Alzheimer, esquizofrenia, epilepsia e tabagismo. Revista de Psiquiatria Clinica, 37(2), 74-80. https://doi.org/10.1590/S0101-60832010000200007.

Viegas Junior, C., Bolzani, V. S., Furlan, M., Fraga, C. A. M., \& Barreiro, E. J. (2004) Protudos naturais como candidatos a fármacos utéis no tratamento do mal de alzheimer. Quimica Nova, 27(4), 631-639. https://doi.org/10.1590/S010040422004000400018.

Yallapu, M. M., Jaggi, M., \& Chauhan, S. C. (2010). B-Cyclodextrin-curcumin self-assembly enhances curcumin delivery in prostate cancer cells. Colloids and Surfaces B: Biointerfaces, 79(1), 113-125. https://doi.org/10.1016/j.colsurfb.2010.03.039.

Ye, F., Lei, D., Wang, S., \& Zhao, G. (2017). Polymeric micelles of octenylsuccinated corn dextrin as vehicles to solubilize curcumin. LWT - Food Science and Technology, 75, 187-194. https://doi.org/10.1016/j.lwt.2016.08.054.

Zhao, C., Cheng, H., Jiang, P., Yao, Y., \& Han, J. (2014). Preparation of lutein-loaded particles for improving solubility and stability by Polyvinylpyrrolidone (PVP) as an emulsion-stabilizer. Food Chemistry, 156, 123-128. https://doi.org/10.1016/j. foodchem.2014.01.086 